Emir. J. Agric. Sci. 2006. 18 (2): 20-28

http://www.cfa.uaeu.ac.ae/research/ejas.htm

\title{
Antioxidant activities of extracts from Combretum hartmannianum and Guiera senegalensis on the oxidative stability of sunflower oil
}

\author{
A. Mariod ${ }^{1}$, B. Matthäus ${ }^{2}$ and I. H. Hussein ${ }^{3}$ \\ ${ }^{1}$ Food Science \& Technology Department, College of Agricultural Studies, Sudan \\ University of Science \& Technology, P.O. Box 71 Khartoum North, Sudan, ${ }^{2}$ Institute for \\ Lipid Research of the Federal Research Center for Nutrition and Food, Piusallee 68/76, \\ 48147 Münster, Germany, ${ }^{3}$ National Oil Processing Research Institute, University of \\ Gezira, P.O. Box 20, Wad Madani Sudan
}

\begin{abstract}
This study was carried out to investigate the total phenolic compounds beside the potential antioxidant activity of Guiera senegalensis and Combretum hartmannianum extracts. The content of total phenolics in the extracts was determined spectrometrically according to the Folin-Ciocalteu procedure and calculated as gallic acid equivalents (GAE). The antioxidative activities by beta-carotene linoleic acid and DPPH methods of extracts from Guiera senegalensis leaves, (GSL), Guiera senegalensis roots (GSR) and Combretum hartmannianum leaves (CHL), were evaluated in sunflower oil in the dark at $70^{\circ} \mathrm{C}$. Total phenolic compounds were 240.1, 275.6 and $253.4 \mathrm{mg} / \mathrm{g}$ respectively. The methanolic extracts of (GSL), (GSR) and (CHL), were markedly effective in inhibiting the oxidation of linoleic acid and subsequent bleaching of ß-carotene in comparison with the control. Plants extracts (500 mg) effectively inhibited the formation of peroxides in sunflower oil and showed the highest antioxidative activity compared with $20 \mathrm{mg}$ BHA. The GSL extract is the most effective followed by the GSR and CHL extract. The antioxidant activity of the extracts measured by DPPH free radical showed high reduction of 50\% DPPH in CHL extract followed by GSR and GSL. Clear differences were found between the control and sunflower oil containing $C$. hartmannianum and $G$. senegalensis extracts or BHA, which decreased and slowed the rate of peroxide formation resulting in lower PVs after $96 \mathrm{~h}$ of storage at $70^{\circ} \mathrm{C}$.
\end{abstract}

Keywords: Antioxidant activity, ß-carotene/linolenic acid, Combretum hartmannianum, 1,1diphenyl-2 picrylhydrazyl (DPPH), Guiera senegalensis, phenolic compounds.

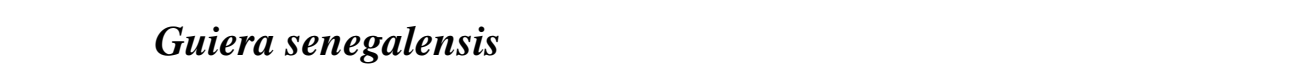

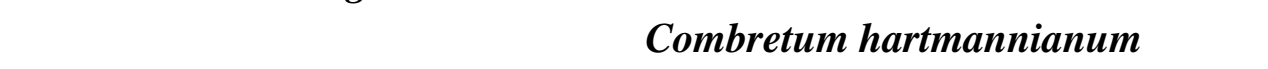

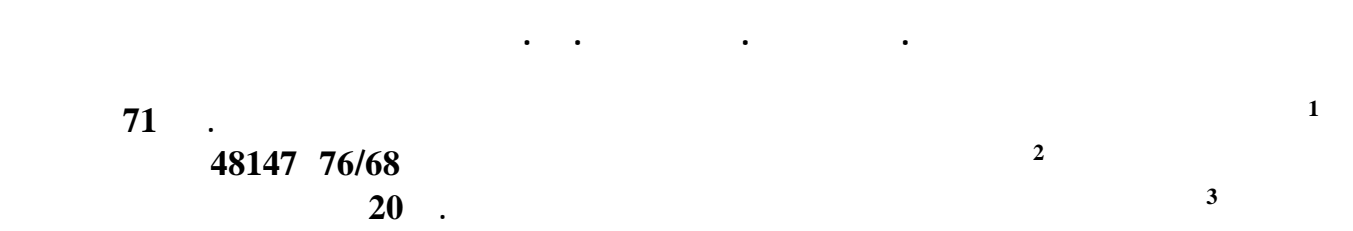

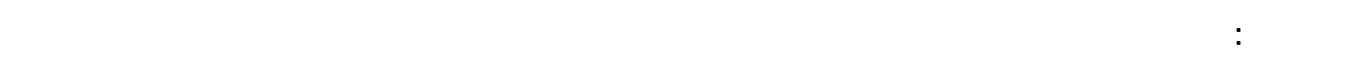

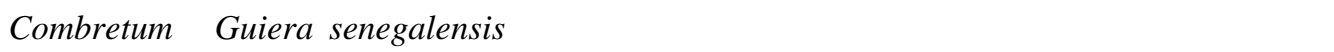

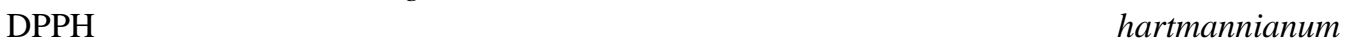

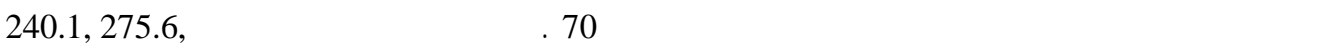

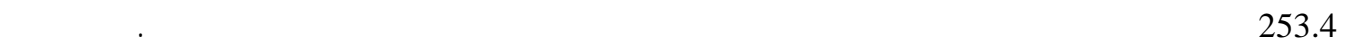

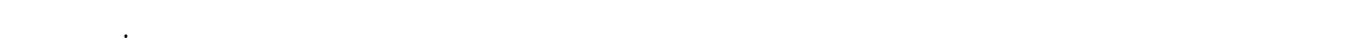

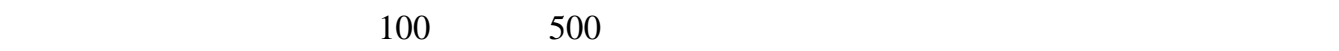

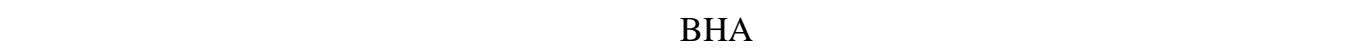

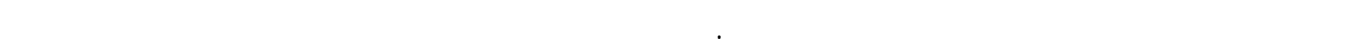

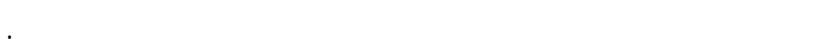

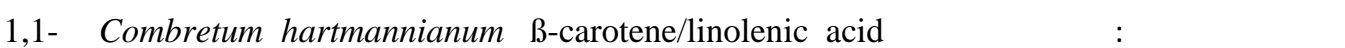
. Guiera senegalensis ،diphenyl-2 picrylhydrazyl (DPPH) 


\section{Introduction}

Plants have been studied as sources of different classes of natural antioxidants. Some plants, grown wild or cultivated, have been exploited commercially for many years (Exarchou et al., 2002). Guiera senegalensis family combretaceae known locally in Sudan as Ghibaish is a shrub, up to $3 \mathrm{~m}$ high, leaves were used for leprosy prevention, the root decoction is used to cure diarrhea, dysentery. Combretum hartmannianum a shrub up to $4 \mathrm{~m}$, as a tree under favorable conditions $10 \mathrm{~m}$ high known locally in Sudan as Habeel. Leaves alternate, shining light green when young, typically rust-colored when mature, leaves were used as an antipyretic, diuretic and for various diseases such as yellow fever, hepatic disorder (Maydell 1990). The methanolic extracts of different parts of Combretum hartmannianum possessed significant activity against the chloroquine-sensitive Plasmodium falciparum strain (El Ghazali et al., 1994). The extracts of the bark and leaves of Combretum hartmannianum exhibited low activity against tuberculosis ( $T . b)$ rhodesiense. Antiparasitic activities have also been described for plants belonging to the family Combretaceae (Benoit et al., 1996; Udem et al., 1996). Furthermore, triterpenes, stilbenes, and methoxylated flavonoids, were reported from the different Combretum species (Jossang et al., 1994; Pettit et al., 2000).

During storage of oils, fats and other fat containing foods, lipid oxidation is still a major cause of food quality deterioration in spite of a wide use of several antioxidants. Antioxidants are added to lipids or foods containing fats to retard the formation of various offflavors. The use of BHA, BHT and TBHQ has been decreasing because it is suspected, but not proven, that they may act as a general consumer rejection of the use of synthetic food additives (Namiki., 1990). Phenolic compounds are regarded as substances derived from the shikimate pathway and phenylpropanoid metabolism and form one of the main classes of secondary metabolites possessing an aromatic ring bearing one or more hydroxy groups (Robards et al., 1999). There is increasing evidence that consumption of a variety of phenolic compounds present in natural foods may lower the risk of serious health disorders because of the antioxidant activity of these compounds (Keli et al., 1996, Hertog et al., 1993). Many studies investigated natural sources of phenolic antioxidants, which could substitute synthetic additives, and also play an important role in preventing many diseases. Special attention is focused on the isolation of natural phenolic antioxidants from inexpensive sources. Recently, interest has increased considerably in finding naturally occurring antioxidants for use in foods or medicinal materials to replace synthetic antioxidants, which are being restricted due to their carcinogenicity. Therefore, the search for preparations of useful natural antioxidants is highly desirable. Among the various plants grown in Sudan, some endemic species are of particular interest because they may be used for the production of raw materials or preparations containing phytochemicals with significant antioxidant capacities and health benefits.

The purpose of this study was to (1) determine the total phenolic compounds of extracts from Guiera senegalensis and Combretum hartmannianum that were growing in the same location and same conditions with respect to their total phenolic content and antioxidant activity to find new potential sources of natural antioxidants; (2) examine the antioxidative activity of extracts from Guiera senegalensis and Combretum hartmannianum in sunflower oil in the dark at $70^{\circ} \mathrm{C}$ and (3) evaluate the relationship between phenolic content and antioxidant activity. 
Emir. J. Agric. Sci. 2006. 18 (2): 20-28

http://www.cfa.uaeu.ac.ae/research/ejas.htm

\section{Materials and methods}

Solvents and Reagents

All solvents used were of analytical grade. $n$-hexane, methanol (HPLC grade), chloroform, BHA, Folin Ciocalteau phenol reagent and polyoxye-thylenesorbitan monopalmitate (Tween 40) were obtained from Merck (Darmstadt, Germany).

Leaves, root of Guiera senegalensis and leaves of Combretum hartmannianum were collected from Ghibaish, Western Sudan. The collected plant parts were sun dried, crushed, and ground by a grinding mill (Petra electric, Burgau, Germany).

\section{Sunflower oil}

Sunflower oil free from additives was used as the substrate for oxidation studies. Refined sunflower oil was obtained from Aldi supermarket Co. (Muenster, Germany). The oil main fatty acids were palmitic acid $5.7 \%$, stearic acid $3.8 \%$, oleic acid $41.3 \%$, linoleic acid $46.3 \%$. The oil peroxide and acid values were $2.02 \mathrm{meq} \mathrm{O}_{2} / \mathrm{kg}$, and $0.36 \mathrm{mg}$ $\mathrm{KOH} / \mathrm{kg}$ oil, respectively.

\section{Phenolic compounds extraction}

The phenolic compounds were extracted from air-dried, coarsely ground leaves, root of Guiera senegalensis and leaves of Combretum hartmannianum, using liquid-liquid extraction according to Silvia et al., (1984). $25 \mathrm{~g}$ of dried grounded material were dissolved in 50 $\mathrm{ml}$ hexane and extracted three times with $30 \mathrm{ml}$ of methanol/water $(60: 40, \mathrm{v} / \mathrm{v})$. The combined extracts were evaporated to dryness in a rotary evaporator at $40^{\circ} \mathrm{C}$.

Determination of the total amount of phenolic compounds

The total amount of phenolic compounds in leaves, root of Guiera senegalensis and leaves of Combretum hartmannianum extract was determined following the method of Silvia et al.,
(1984). Dried samples (5 mg) and gallic acid as standard were diluted in $1 \mathrm{ml}$ of 60:40 acidified methanol/water $(0.3 \%$ $\mathrm{HCl}$ ). Test solutions (samples or standards) of $100 \mu \mathrm{L}$ were added to 2.0 $\mathrm{ml}$ of $2 \% \mathrm{Na}_{2} \mathrm{CO}_{3}$. After 2 min., $100 \mu \mathrm{L}$ of $50 \%$ Folin-Ciocalteau reagent were added and allowed to stand at room temperature for $30 \mathrm{~min}$. A blank consisted of all reagents and solvents without extracts or standard were prepared. Absorbance was measured against the blank at $750 \mathrm{~nm}$ by an U-2000 Spectrophotometer (Hitachi, LTD. Tokyo, Japan). The standard solution was prepared from gallic acid in concentrations of $0.05 \mathrm{mg} / \mathrm{ml}$ to 0.4 $\mathrm{mg} / \mathrm{ml}$. The concentration of phenolic compounds in the extracts was calculated from a calibration curve of the standard, and expressed as gallic acid equivalents (GAE).

Determination of the antioxidant activity $ß$-carotene-linoleic acid assay

The antioxidant activity of the leaves, root of Guiera senegalensis and leaves of Combretum hartmannianum extracts were evaluated using the ß-carotenelinoleic acid assay (Amarowics et al., 2003). Approximately $2 \mathrm{mg} ß$-carotene were dissolved in $10 \mathrm{ml}$ of chloroform. One milliliter of this solution was pipetted into a round-bottom flask. After removal of chloroform using a rotary evaporator, $20 \mathrm{mg}$ of linoleic acid, 200 $\mathrm{mg}$ of Tween 40, and $50 \mathrm{ml}$ of distilled water were added to the flask with vigorous stirring. Aliquots $(5 \mathrm{ml})$ of the prepared emulsion were transferred to a series of tubes containing $2 \mathrm{mg}$ of each extract or $0.2 \mathrm{mg}$ of butylated hydroxyanisole (BHA). The samples were put in a water bath at $50{ }^{\circ} \mathrm{C}$ over a period of $120 \mathrm{~min}$. Subsequently; absorbance was read at $470 \mathrm{~nm}$, every 15 min. All samples were assayed in duplicate. 
DPPH method

The antioxidant activity of the leaves, root of Guiera senegalensis and leaves of Combretum hartmannianum phenolic extracts was measured following Gordon et al., (2001) in terms of hydrogen donating or radical scavenging ability using the stable radical 1,1-Diphenyl- $\beta$ picrylhydrazyl (DPPH). A methanolic solution (25, 50, 100, 200, 300, 400, and $500 \mu \mathrm{L}$ ) of the phenolic compounds extracted from the two plants parts was placed in a cuvette and $0.5 \mathrm{ml}$ of a methanolic solution of DPPH (50 mg $\mathrm{DPPH} / 100 \mathrm{ml} \mathrm{MeOH}$ ) was added. After 30 min incubation in darkness and at ambient temperature $\left(23^{\circ} \mathrm{C}\right)$, the resultant absorbance was recorded at $515 \mathrm{~nm}$. The decrease in absorbance at $515 \mathrm{~nm}$ was determined with a Hitachi spectrophotometer (Hitachi, LTD. Tokyo, Japan), the absorbance of the DPPH radical without antioxidant, i.e the control was measured. All determinations were performed in duplicate.

Oxidation of Sunflower oil using $G$. senegalensis and $C$. hartmannianum extracts

The dried extracts of leaves, root of Guiera senegalensis and Combretum leaves of hartmannianum were applied to $100 \mathrm{~g}$ of sunflower oil at levels of 500 , and $1000 \mathrm{mg}$ to examine their antioxidative activity. BHA was used for comparison at a level of $200 \mathrm{ppm}$. The dried extracts as well as the synthetic antioxidant were mixed with a minimum amount of absolute methanol in an ultrasonic water bath (Bandelin electronic, Berlin, Germany) and added to $100 \mathrm{~g}$ oil before mixing again for 10 min. A control sample was prepared by using the same amount of methanol used to dissolve BHA and the extracts (Moure et al., 2000). The samples and control were stored into 250-ml Erlenmeyer flasks in ambient air and were oxidized at $70{ }^{\circ} \mathrm{C}$ in the dark in a shaker water bath (Kottermann, Germany). The peroxide value (PV) and the inhibition of the oil oxidation (IO) were used as indicators for the primary oxidation of the sunflower oil. The PVs were determined every 4, 24 , and 72 and 96 hours. IO $=(100 \%-$ (PV increase of samples/PV increase of control) X 100\% (Duh et al., 1997).

\section{Statistical Analysis}

Each value is a mean of three replications. Values of different parameters were expressed as the mean \pm standard deviation (mean \pm SD).

\section{Results and discussion}

The phenolic compound of Guiera senegalensis leaves (GSL) Guiera senegalensis roots (GSR) and Combretum hartmannianum leaves (CHL) were extracted. In Table 1 the content of the total phenolic compounds of the different extracts, determined according to the Folin-Ciocalteau method is shown. The results showed that GSR has the highest amount of total phenolic compounds followed by CHL and GSL. It becomes clear that the total phenolic compounds were not related to the total extractable materials. The antioxidant activity of phenolic compounds may result from the neutralization of free radicals initiating oxidation processes or from the termination of radical chain reactions. For this reason, two different methods have been used for the determination of the antioxidant activity of the extracts: Inhibition of $\beta$-carotene co-oxidation in a linoleate model system and DPPH free radical scavenging. Figure1 revealed the decrease of absorbance of $ß$-carotene in the presence of different plant extracts in the coupled system of ß-carotene and linoleic acid at $50^{\circ} \mathrm{C}$. It showed that the control sample without addition of extract solution oxidized most rapidly. The methanolic extracts of GSL, GSR, and CHL were markedly effective in inhibiting the oxidation of linoleic acid and subsequent 
bleaching of $ß$-carotene in comparison with the control. The GSL extract is the most effective followed by the GSR and CHL extract; there is no correlation between the level of phenolic compound in the extracts and their antioxidant activity.

The $\mathrm{DPPH}^{\bullet}$ radical is considered to be a model of a stable lipophilic radical. A chain reaction in lipophilic radicals was initiated by the lipid autoxidation. Antioxidants react with $\mathrm{DPPH}^{\bullet}$, reducing a number $\mathrm{DPPH}^{\bullet}$ molecules equal to the number of their available hydroxyl groups. Therefore, the absorption at 515 $\mathrm{nm}$ was proportional to the amount of residual $\mathrm{DPPH}^{\bullet}$ (Xu et al (2005). In Figure2 the decrease in absorbance of the $\mathrm{DPPH}$ radical $\left(\mathrm{DPPH}^{\circ}\right)$ due to its reduction by different plant extracts is illustrated. Absorbance decreases as a result of a color change from purple to yellow as the radical is scavenged by antioxidants through donation of hydrogen to form the stable DPPH-H. The more rapidly the absorbance decreases, the more potent the antioxidant activity of the compound in terms of hydrogen donating ability (Yen and Duh, 1994). The antioxidant activity determined by the DPPH method revealed that the $\mathrm{CHL}$ extract had the highest antioxidant activity on DPPH free radicals followed by the GSR and GSL extract Figure2; only $0.04 \mathrm{mg}$ extract of CHL was necessary to reach $50 \%$ loss in $\mathrm{DPPH}$ while in case of GSR and GSL $0.08 \mathrm{mg}$ were used.

Effect of plant extracts on sunflower oil oxidation

The effects of individual plant extracts on the oxidative stability of traditional sunflower oil, which is very rich in linoleic fatty acid, were evaluated by measuring the formation of peroxide value at $70^{\circ} \mathrm{C}$. This temperature was used, because at higher temperatures the peroxides will decompose very fast (Duh and Yen 1997). The PV of sunflower oil (control) with and without GSL, GSR, CHL or BHA showed a linear increase. As demonstrated in Figures 3 and 4. A maximum PV value of 58.5 meq $\mathrm{O}_{2} / \mathrm{kg}$ was reached after 96 hours of storage in the control without addition of extract or BHA. A clear significant differences ( $P$ $<0.05$ ) were found between the control and sunflower oil containing GSL, GSR, CHL extracts or BHA, which decreased and slowed the rate of peroxide formation resulting in lower PVs after $96 \mathrm{~h}$ of storage at $70{ }^{\circ} \mathrm{C}$. The PVs of sunflower oil containing $500 \mathrm{mg}$ of GSL, GSR, CHL or $20 \mathrm{mg}$ BHA were found as 9.6, 11.9, 12.5 and 23.0 meq $\mathrm{O}_{2} / \mathrm{kg}$, respectively (Fig.3). These samples showed an inhibition of oxidation (IO) after 96 hours of storage of 91.2, 85.8, 84.8, and $67.3 \%$ respectively, in comparison to a control. GSL showed the highest activity, followed by GSR and CHL which were higher than the effect of BHA.

The PVs of sunflower oil containing $1000 \mathrm{mg}$ GSL, GSR, CHL or $20 \mathrm{mg}$ BHA were found as $47.0,21.5,23.5$, and 23.0 meq $\mathrm{O}_{2} / \mathrm{kg}$, respectively (Fig.4). These samples showed an inhibition of oxidation (IO) after 96 hours of storage of 18.3, 67.0, 63.3, and $67.3 \%$ respectively, in comparison to a control GSR showed the highest activity, but all the extracts showed less inhibition of oxidation than BHA. The addition of $1000 \mathrm{mg}$ from the extracts seems to be not effective enough in oil oxidation inhibition. Our results suggest that these plants extracts act as general antioxidants inhibiting the generation of peroxides regardless of the amount of the plant extracts. It seems too difficult to explain this, however, the antioxidant activities of plant antioxidants have been attributed to various mechanisms, among which are prevention of chain initiation, binding of transition metal ion catalysts, decomposition of peroxides, prevention of continued hydrogen abstraction, and radical scavenging. From this it can be 
assumed that $500 \mathrm{mg}$ extracts added had a remarkable antioxidative activity to reduce oil degradation, therefore the development of rancidity in sunflower oil was suppressed owing to the presence of these antioxidant compounds in $G$. senegalensis and $C$. hartmannianum extract. The obtained results suggest the necessity of performing more detailed investigations to explain the reasons of antioxidative ability changes of $G$. senegalensis and $C$. hartmannianum different extracts.

Table 1. Total extractable compounds and total phenolic compounds in C. hartmannianum leaves (CHL), G. senegalensis leaves, (GSL), and G. senegalensis roots (GSR) extracts.

\begin{tabular}{lll}
\hline Sample & Total extractable $(\mathbf{g} / \mathbf{g})$ & Total phenolic $(\mathbf{m g} / \mathbf{g})$ \\
\hline CHL & $8.2695 \pm 0.03$ & $253.4 \pm 0.1$ \\
GSR & $0.9807 \pm 0.02$ & $275.6 \pm 0.1$ \\
GSL & $5.0900 \pm 0.1$ & $240.1 \pm 0.05$ \\
\hline
\end{tabular}

Total phenolic compounds in the dried extracts as gallic acid equivalent (GAE). Data are means of triplicate results and mean value \pm standard deviation (SD) reported

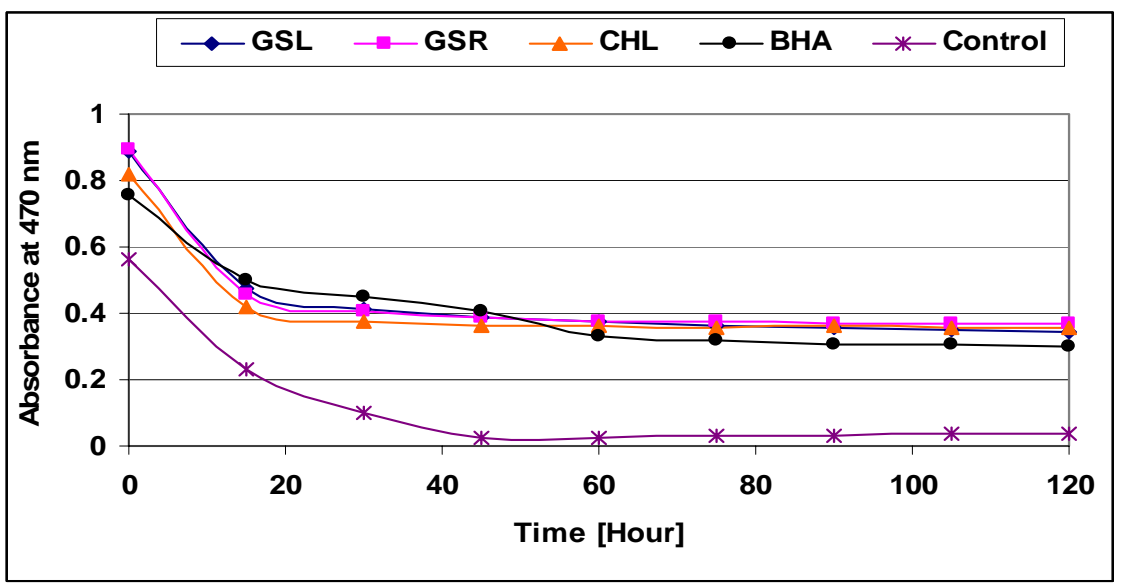

Figure 1. Antioxidant activity of extracts obtained from C. hartmannianum leaves (CHL), G. senegalensis leaves, (GSL), and G. senegalensis roots (GSR) measured by the bleaching time of $\beta$-carotene at $50 \mathrm{C}$.

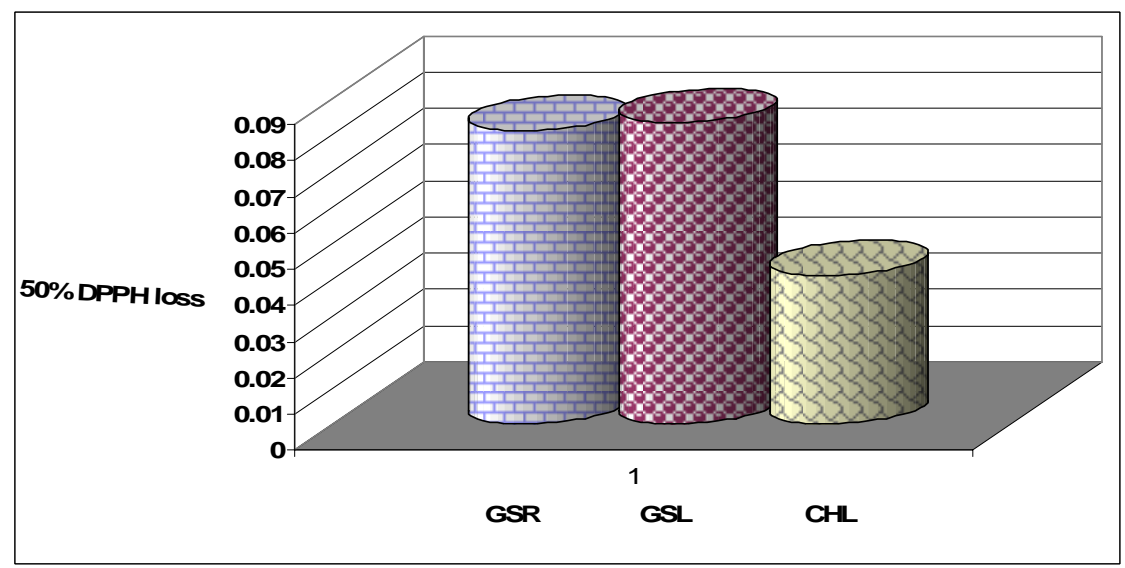


Emir. J. Agric. Sci. 2006. 18 (2): 20-28

http://www.cfa.uaeu.ac.ae/research/ejas.htm

Figure 2. Effect of $C$. hartmannianum, and G. senegalensis extracts on DPPH free radical (expressed as mg extract allowing reduction of $50 \%$ DPPH).

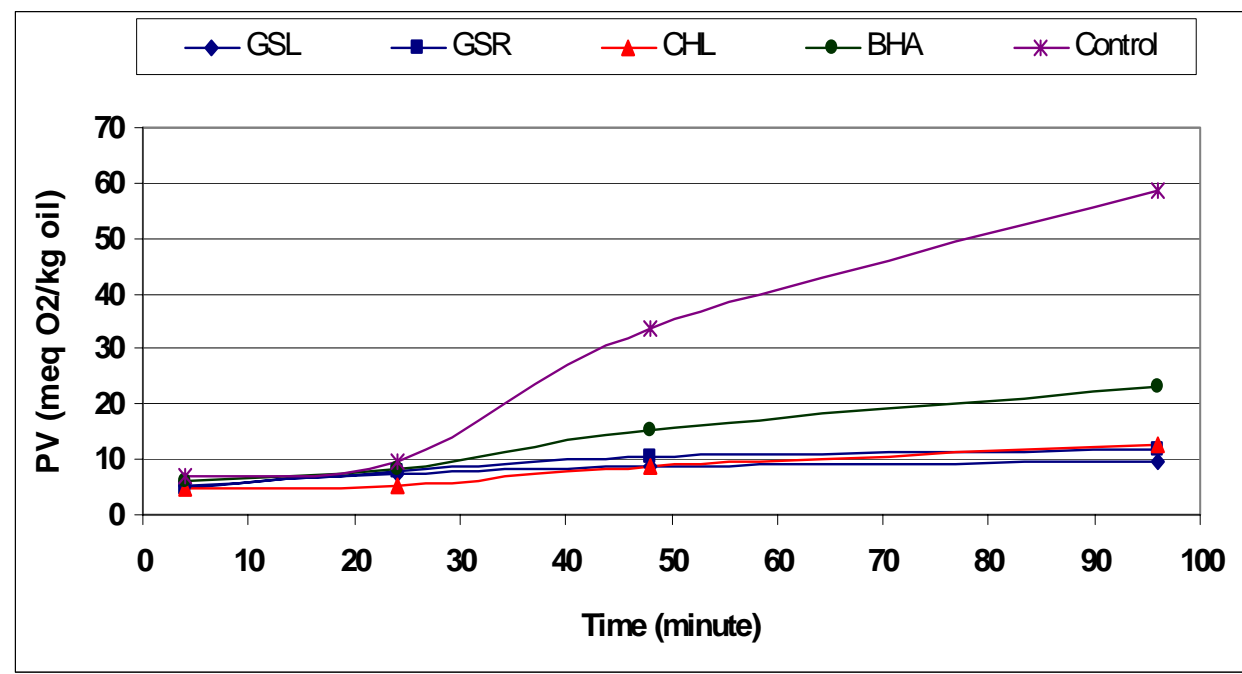

Figure 3. Oxidation of sunflower oil treated with $C$. hartmannianum, and $G$. senegalensis extract $500 \mathrm{mg} / 100 \mathrm{~g}$ oil during storage at $70^{\circ} \mathrm{C}$.

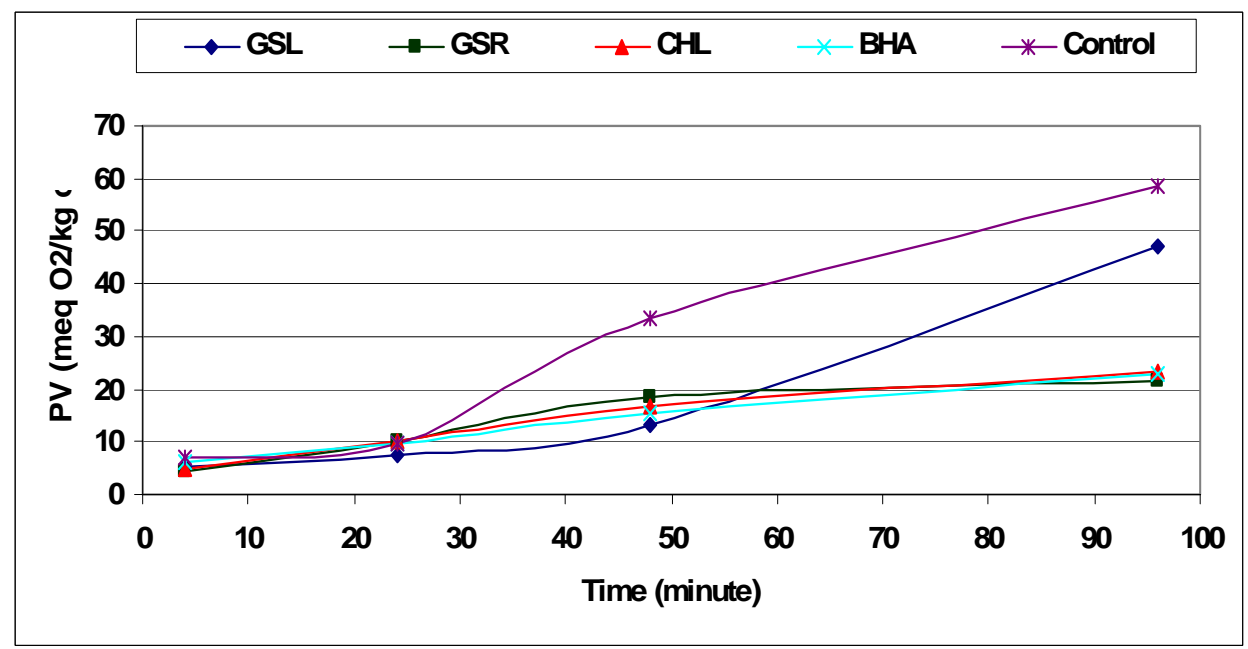

Figure 4. Oxidation of sunflower oil treated with $C$. hartmannianum, and $G$. senegalensis $1000 \mathrm{mg} / 100 \mathrm{~g}$ oil during storage at $70^{\circ} \mathrm{C}$.

\section{Conclusions}

The current study showed that phenolic compounds that are present in the extracts of $G$. senegalensis and $C$. hartmannianum had significant antioxidant activity relative to BHA, a commercial antioxidant in foods. The study showed that $G$. senegalensis root extract and $C$. hartmannianum leaves extract has the highest amount of total phenolic compounds followed by $G$. senegalensis leaves extract. It can be concluded that $G$. senegalensis and $C$. hartmannianum extracts at concentrations of $0.5 \% \quad(\mathrm{w} / \mathrm{w})$ were effective in stabilizing sunflower oil during storage at $70{ }^{\circ} \mathrm{C}$. These samples showed an inhibition of oxidation (IO) after 96 hours of storage in comparison to a control. This makes a strong impact on expanding the investigations of antioxidant constituents present in these plant 
extracts and being used to protect oils against oxidation.

\section{Acknowledgements}

The first author thanks the National Oilseed Processing and Research Institute (NOPRI), University of Gezira, Sudan and German Academic Exchange Service (DAAD) for their financial support.

\section{References}

Ali, H., G. M. Koenig, S. A. Khalid, A. D. Wright and R. Kaminsky. 2002. Evaluation of selected Sudanese medicinal plants for their in vitro activity against hemoflagellates, selected bacteria, HIV-1-RT and tyrosine kinase inhibitory, and for cytotoxicity. Journal of Ethnopharmacology 83:219-228.

Amarowics, R., M. Karamac and F. Shahidi. 2003. Antioxidant activity of phenolic fractions of lentil (Lens culinaris). J. Food Lipids 10:1-10.

Benoit, V. F., A. Valentin, Y. Pelissier, F. Diafouka, C. Marion, D. KoneBamba, M. Kone, M. Mallie, A. Yapo and J. M. Bastide. 1996. In vitro anti malarial activity of vegetal extracts used in West African traditional medicine. American Journal of Tropical Medicine and Hygiene. 54 (1):67-71.

Duh, P. D., and Y. C. Yen. 1997. Antioxidant efficacy of methanolic extracts of peanut hulls in soybean and peanut oils. J. Am. Oil Chem. Soc. 74:745-748.

El Ghazali, G. B., M. S. El Tohami and A. B. El Egami. 1994. Medicinal Plants of the Sudan. Part III. Medicinal Plants of the White Nile Province. Khartoum University Press, Sudan.
Exarchou, V., N. Nenadis, M. Tsimidou, I. P. Gerothanassis, A. Troganis and D. Boskou. 2002. Antioxidant Activities and Phenolic Composition of Extracts from Greek Oregano, Greek Sage, and Summer Savory J. Agric. Food Chem. 50:5294-5299.

Gordon, M. H., F. Paiva-Martins and M. Almeida. 2001. Antioxidant activity of hydroxytyrosol acetate compared with that of other olive oil polyphenols J. Agric. Food Chem. 49:2480-2485.

Hertog, M. G. L., E. J. M. Feskens, P. C. H. Hollman, M. B. Katan and D. Kromhout. 1993. Dietary antioxidant flavonoids and risk of coronary heart diseases -The Zutphen Elderly study. Lancet. 342:1007-1011.

Jossang, A., J. L. Pousset, B. Bodo. 1994. Combreglutinin, hydrolysable tannin from Combretum glutinosum. Journal of Natural Products 57(6):732-737.

Keli, S. O., M. G. L. Hertog, E. J. M. Feskens and D. Kromhout. 1996. Dietary flavonoids, antioxidant vitamins and incidence of strokesThe Zutphen study. Arch. Intern. Med. 156:637- 642.

Maydell, H. J. V. 1990. Trees and Shrubs of the Sahel. Their Characteristics and Uses, (GTZ) GmbH, Germany.

Namiki, M. 1990. Anti-oxidants/antimutagenes in food critical. Reviews in Food Science and Nutrition 29: 273.

Pettit, G. R., M. P. Grealish, D. L. Herald, M. R. Boyd, E. Hamel and R. K. Pettit, 2000. Antineoplastic agents 443: synthesis of the cancer cell growth inhibitor hydroxylphenstatin and its sodium diphosphate 
Emir. J. Agric. Sci. 2006. 18 (2): 20-28

http://www.cfa.uaeu.ac.ae/research/ejas.htm

prodrug. Journal of Medicinal Chemistry 43(14):2731-2737.

Robards K., P. D. Prenzler, G. Tucker, P. Swatsitang and W. Glovera. 1999. Phenolic compounds and their role in oxidative processes in fruits. Food Chemistry 66:401-436.

Silvia M. T., E. E. Miller and D. E. Pratt. 1984. Chia seeds as a source of natural lipid antioxidants. J. Am. Oil Chem. Soc. 61:928-931.

Udem, S. C., I. Madubunyi, I. U. Asuzu and A. M. Anika. 1996. The trypanocidal action of the root extract of Combretum dolichopetalum. Fitote-rapia 67(1):31-37.

Xu, J. and S. Chen Q. Hu. 2005. Antioxidant activity of brown pigment and extracts from black sesame seed (Sesamum indicum L.) Food Chemistry 91:7-83.

Yen, G. C. and P. D. Duh. 1994. Scavenging effect of methanolic extracts of peanut hulls on freeradical and active-oxygen species. J. Agric. Food Chem. 42:629-632. 